\title{
Fiber surfaces from alternating states
}

\author{
DARLAN GIRÃO \\ Jỗo NOGUEIRA \\ ANTÓNIO SALGUEIRO
}

\begin{abstract}
In this paper we define alternating Kauffman states of links and we characterize when the induced state surface is a fiber. In addition, we give a different proof of a similar theorem of Futer, Kalfagianni and Purcell on homogeneous states.
\end{abstract}

57M25; 57M15

\section{Introduction}

Let $K$ be a link in $S^{3}$. We say the link $K$ is fibered if $S^{3}-K$ has the structure of a surface bundle over the circle, ie if there exists a Seifert surface $S$ such that $S^{3}-K \cong(S \times[0,1]) / \varphi$, where $\varphi$ is a homeomorphism of $S$. In this case we abuse terminology and say $S$ is a fiber for $K$. The study of the fibration of link complements has been a very active line of research in low dimensional topology. In the next two paragraphs we highlight some of the work in this area.

In the early 1960s, Murasugi [7] proved that an alternating link is fibered if and only if its reduced Alexander polynomial is monic. Stallings [8] proved that a link $K$ is fibered if and only if $\pi_{1}\left(S^{3}-K\right)$ contains a finitely generated normal subgroup whose quotient is $\mathbb{Z}$. Stallings' result is very general, but hard to verify, even if we restrict to particular families of links. In [6], Harer showed how to construct all fibered knots and links using Stallings' operations introduced in [8]. However, deciding whether or not a link $K$ is fibered is, in general, a hard problem. Goodman and Tavares [5] showed that under simple conditions imposed on certain Seifert surfaces for pretzel links, it is possible to decide whether or not these surfaces are fibers. In [3], Gabai proved that if a Seifert surface $S$ can be decomposed as the Murasugi sum of surfaces $S_{1}, \ldots, S_{n}$, then $S$ is a fiber if and only if each of the surfaces $S_{i}$ is a fiber (refer to Theorem 3 ).

Very recently Futer, Kalfagianni and Purcell [2] introduced a new method for deciding whether some Seifert surfaces are fibers. From a particular diagram of the link, they construct an associated surface (called a state surface) and a certain graph. If the state is homogeneous, they show that this surface is a fiber if and only if the corresponding 
graph is a tree (Theorem 2 below). Later, Futer [1] gave a different, much simpler proof of this result. Based on the work of Gabai [3] and Stallings [8], the first author [4] studied fibration of state surfaces of augmented links. This paper is concerned with the study of another class of state surfaces, which we now describe.

Given a diagram $D$ of a link $L$ we can construct a collection of disjoint disks connected by a twisted band at each crossing. We thus obtain a surface whose boundary is the link $L$. The disks and bands are defined by how we split the crossings in the diagram of $L$. At each crossing there are two choices of resolutions for the split: an $A$-resolution or a $B$-resolution, as presented in Figure 1.
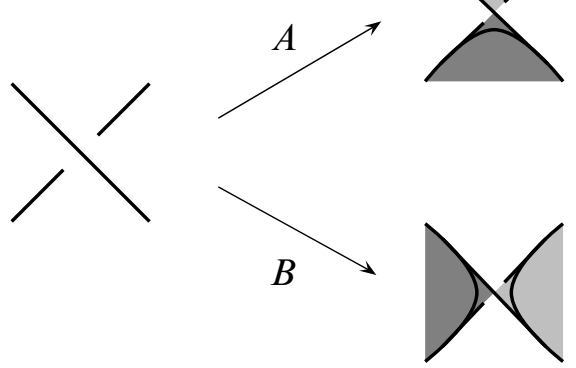
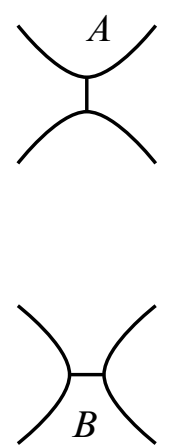

Figure 1: The two choices of resolutions for the split of a crossing

A Kauffman state $\sigma$ of a link diagram $D$ is a choice of resolution for each crossing of $D$. The resulting surface $S_{\sigma}$ is called the state surface of $\sigma$. The boundaries of the disks induce a decomposition of the plane into connected components that we call regions. The well-known Seifert surface of an oriented diagram of a link is a particular case of a state surface, where the resolution of each crossing is defined by the orientation. It has been of interest to identify fibered knots and their fibers. We want to understand when a state surface is a fiber. Futer, Kalfagianni and Purcell [2] studied this for homogeneous states, that is, when all resolutions of the diagram in each region are the same. One of their results was Theorem 2, of which Futer [1] later gave an alternate, simpler proof. In this paper we provide a new approach for this theorem and we prove a similar result for a different type of Kauffman states, as in the next definition.

Definition 1 A Kauffman state $\sigma$ is said to be alternating when for each circle defined by $\sigma$, with a choice of orientation on its boundary, if two consecutive crossings attached to it in the same region have the same resolution then they are adjacent to the same circles defined by $\sigma$. 
Before we present our main result, we associate two graphs to each state of a link diagram. The state graph $G_{\sigma}$ has one vertex for each disk and one edge for each band defined by $\sigma$. We label the edges by the resolution of the respective crossings. The reduced graph $G_{\sigma}^{\prime}$ is obtained from $G_{\sigma}$ by eliminating duplicated edges, with the same label, between two vertices. From the state surface $S_{\sigma}$ we define also a reduced surface $S_{\sigma}^{\prime}$ by cutting duplicated bands with the same label attached to the same pair of disks. We note that the graphs $G_{\sigma}$ and $G_{\sigma}^{\prime}$ are not abstract graphs but are instead embedded in the surfaces $S_{\sigma}$ and $S_{\sigma}^{\prime}$ as their spines. An inner cycle, of the state graph or a reduced version of it, is an innermost cycle in a certain region. Our main result is the following.

Theorem 1 Let $\sigma$ be an alternating state of a link diagram $D_{L}$. Then $E(L)$ fibers over the circle with fiber $S_{\sigma}$ if and only if the reduced graph $G_{\sigma}^{\prime}$ is a tree.

The next examples illustrate that the classes of link diagrams in Theorems 1 and 2 are distinct. Certain states can be both homogeneous and alternating, as for example the Seifert state of the figure-eight knot as in Figure 2.
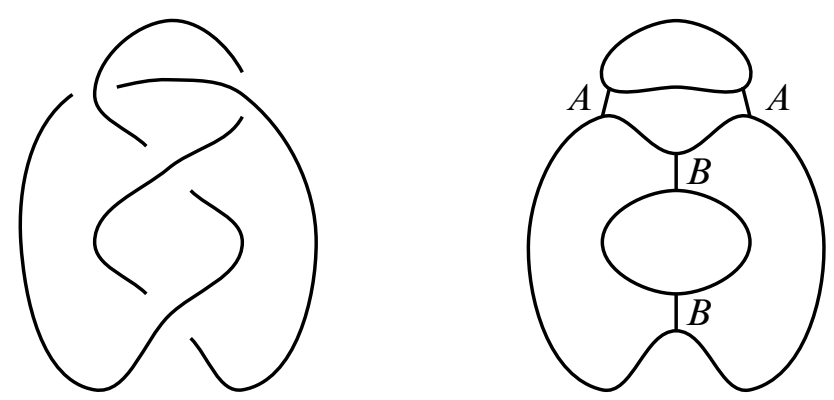

Figure 2: The Seifert state of this figure-eight knot diagram is a fiber by Theorems 1 and 2.

But in general a state isn't both homogeneous and alternating. For instance, in Figure 3 the Seifert state is alternating and not homogeneous.

Furthermore, in the example shown in Figure 4, the Seifert state is homogeneous but not alternating.

The reduced graph of the state in the examples of Figures 2, 3 and 4 is a tree, so in these particular cases the state surface is a fiber. We notice that if $G_{\sigma}$ has edges with different labels between the same pair of vertices then $G_{\sigma}^{\prime}$ is not a tree and, by Theorem $1, S_{\sigma}$ is not a fiber.

In Section 2 we prove this theorem using Murasugi sums and results of Gabai on knot fibration. In Section 3 we give a different, homological proof of the following theorem 

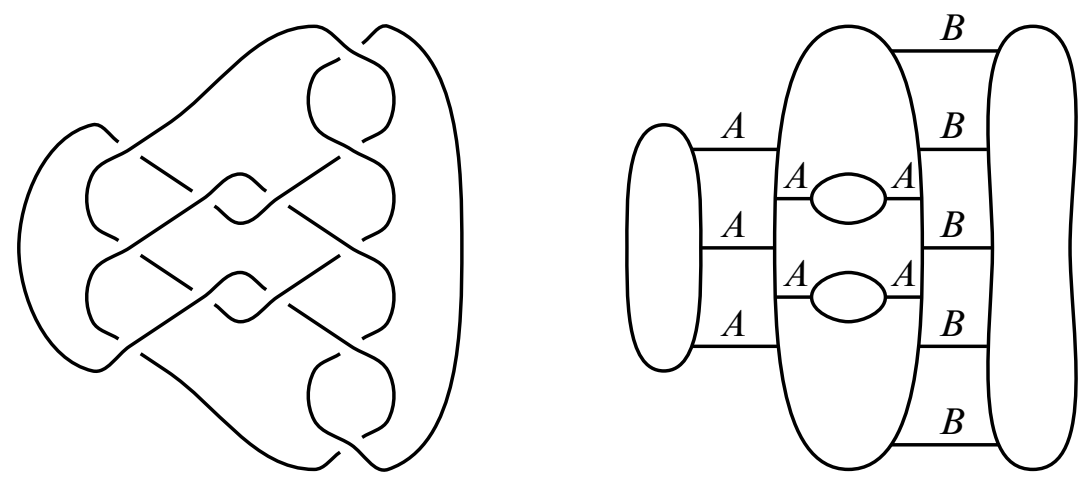

Figure 3: The knot $12 n 0328$ is prime, the Seifert state of this diagram is alternating and not homogeneous, and the corresponding state surface is a fiber by Theorem 1 .
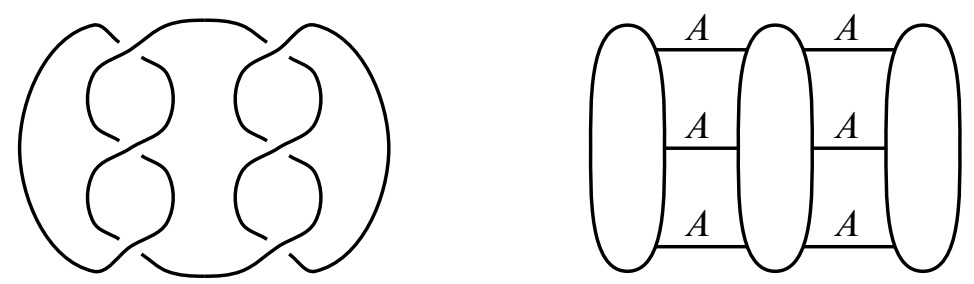

Figure 4: The Seifert state of this granny knot diagram is homogeneous and not alternating, and the corresponding state surface is a fiber by Theorem 2 .

of Futer, Kalfagianni and Purcell [2] on homogeneous states. The techniques we use in our proof are similar to the ones in the paper [4] by the first author, where he studies the fibration of augmented link complements.

Theorem 2 Let $\sigma$ be a homogeneous state of a link diagram $D_{L}$. Then $E(L)$ fibers over the circle with fiber $S_{\sigma}$ if and only if the reduced graph $G_{\sigma}^{\prime}$ is a tree.

\section{Fibers from alternating states}

For this section we use a specific concept of graph decomposition: We say that two vertices $v$ and $w$ decompose a graph $G$ into components $G_{1}, \ldots, G_{k}$ if $G=G_{1} \cup$ $\cdots \cup G_{k}$ and $G_{i} \cap G_{j} \subseteq\{v, w\}$ for $i \neq j$. We also make use of the following theorem by Gabai [3] on Murasugi sum and knot fibration.

Definition 2 We say that the oriented surface $T$ in $S^{3}$ with boundary $L$ is the Murasugi sum of the two oriented surfaces $T_{1}$ and $T_{2}$ with boundaries $L_{1}$ and $L_{2}$ if 
there exists a 2 -sphere $S$ in $S^{3}$ bounding balls $B_{1}$ and $B_{2}$ with $T_{i} \subset B_{i}$ for $i=1,2$, such that $T=T_{1} \cup T_{2}$ and $T_{1} \cap T_{2}=D$, where $D$ is a $2 n$-sided disk contained in $S$.

Theorem 3 (Gabai) Let $T \subset S^{3}$ with $\partial T=L$ be a Murasugi sum of oriented surfaces $T_{i} \subset S^{3}$ with $\partial T_{i}=L_{i}$ for $i=1,2$. Then $S^{3}-L$ is fibered with fiber $T$ if and only if $S^{3}-L_{i}$ is fibered with fiber $T_{i}$ for $i=1,2$.

With the following lemma we are able to prove that we neither lose fibration information by working with the reduced state graph nor with graph decomposition.

Lemma 1 Let $G_{\sigma}$ be a state graph and suppose there are two vertices, $v$ and $w$, adjacent by the edge $X$, that decompose $G_{\sigma}$ into connected components $X, H_{1}, H_{2}$, $\ldots, H_{k}$. (See Figure 5.) Consider also the state surface $S_{i}$ induced by $\sigma$ and the subgraph $X \cup H_{i}$ of $G_{\sigma}, i=1, \ldots, k$. Then $S_{\sigma}$ is a fiber if and only if each surface $S_{1}, \ldots, S_{k}$ is a fiber with respect to its boundary.

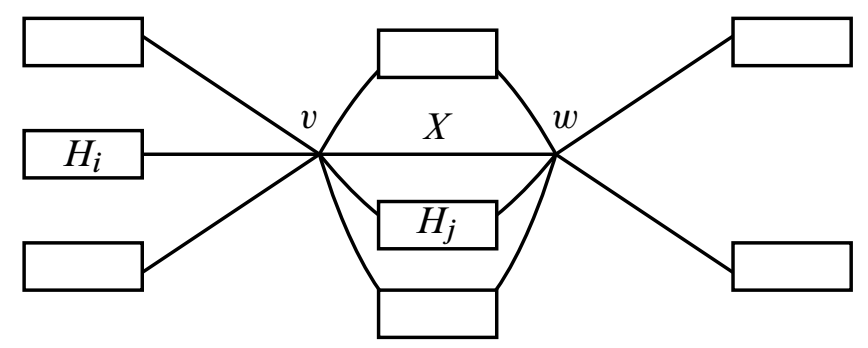

Figure 5: Representation of the decomposition of $G_{\sigma}$ by $v \cup w$

Proof We start by proving that $S_{\sigma}$ is a Murasugi sum of the surfaces $S_{1}, \ldots, S_{k}$. Consider one of the connected components $H_{l}$. If $H_{l}$ contains only one of the vertices $v$ or $w$, then using the disk associated to this vertex and $X$ we can decompose $S_{l}$ from $S_{\sigma}$ by a Murasugi sum. (See Figure 6.) Notice that $S_{l}$ is also the state surface of $H_{l}$, since $X$ contains a terminal vertex in $X \cup H_{l}$.

Let us assume now that $H_{l}$ contains $v \cup w$. Suppose, without loss of generality, that $H_{l}$ is innermost with respect to $X$, ie there is no other component between $H_{l}$ and $X$ in the state graph. We can decompose $S_{l}$ from $S_{\sigma}$ by a Murasugi sum as depicted in Figure 7.

Repeating this procedure with subsequent innermost components we obtain the claimed Murasugi sum decomposition. Therefore, by Theorem 3, $S_{\sigma}$ is a fiber if and only if each surface $S_{l}, \ldots, S_{k}$ is a fiber with respect to its boundary. 


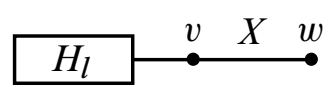

(a)

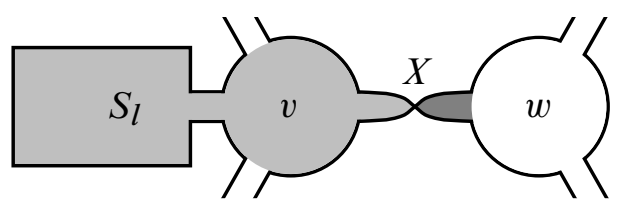

(b)

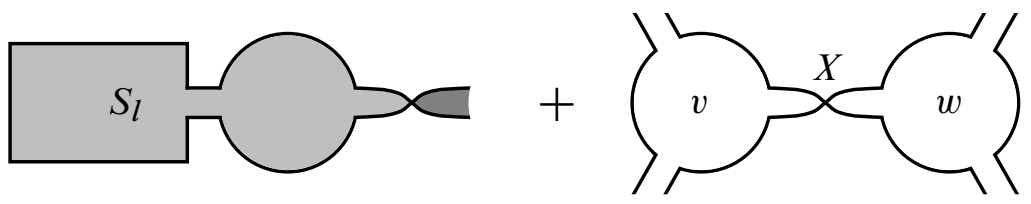

(c)

Figure 6: When $H_{l}$ is adjacent to only $v$ (a), there is a decomposition of $S_{\sigma}$ (b), by $v \cup w$ as a Murasugi sum (c).

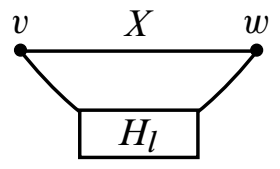

(a)

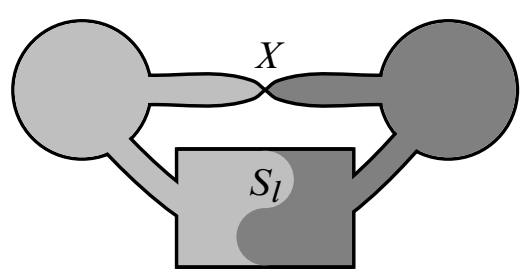

$+$

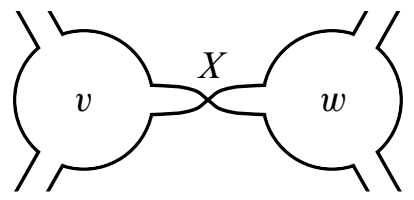

(c)

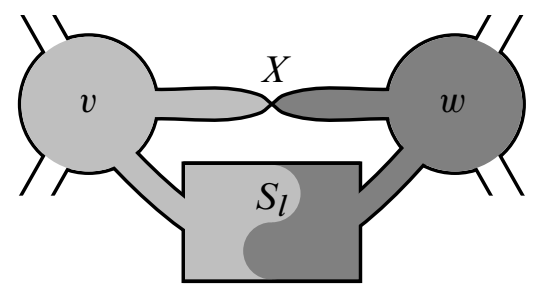

(b)

Figure 7: When $H_{l}$ is adjacent to both $v$ and $w$ (a), there is a decomposition of $S_{\sigma}$ (b), by $v \cup w$ as a Murasugi sum (c).

A particular case of this lemma is when two vertices $v$ and $w$ are adjacent by multiple edges. Take two such edges and suppose $X$ and $Y$ are their labels (we also represent the edges by these letters). Decomposing the graph $G_{\sigma}$ as in the lemma, one of the components obtained corresponds to the edge $Y$. It is not hard to see that the state surface induced by the subgraph $X \cup Y$ is either a Hopf band (when edges have the 
same label) or an untwisted annulus (when edges have different labels). It is well known that the Hopf band is a fiber for the Hopf link in its boundary, and that the untwisted annulus is not a fiber for the unlink in its boundary. For example, a straightforward proof of these facts follows from Theorem 4. This observation lead us to the following corollary.

Corollary 1 Let $G_{\sigma}$ be a state graph and suppose there are vertices $v$ and $w$ adjacent by two edges $X$ and $Y$. If the edges have different labels then the surface $S_{\sigma}$ is not a fiber. If the edges have the same label then $S_{\sigma}$ is a fiber if and only if the state surface induced by the subgraph obtained by removing the edge $Y$ is a fiber.

Proof Decomposing the edge $Y$ from the graph as in Lemma 1, the surface induced by $X \cup Y$ is either an untwisted annulus or a Hopf band. By the observation above and Theorem 3 , in the former case the surface $S_{\sigma}$ is not a fiber; in the latter case $S_{\sigma}$ is a fiber if and only if the remaining Murasugi summands are fibers, that is, if the surface induced by the subgraph obtained by removing the edge $Y$ is a fiber.

Remark 1 In light of Corollary 1 we assume from now on that the state graph $G_{\sigma}$ has no edges with different labels adjacent to the same pair of vertices.

Corollary 1 explains why we do not loose fibering information by passing to the reduced graph $G_{\sigma}^{\prime}$.

Corollary 2 Let $L$ be a link and $\sigma$ a state for a diagram $D_{L}$ of $L$. Let $S_{\sigma}^{\prime}$ be the state surface associated to the reduced graph $G_{\sigma}^{\prime}$ and let $L^{\prime}$ the boundary of $S_{\sigma}^{\prime}$. Then the link $L^{\prime}$ is fibered by $S_{\sigma}^{\prime}$ if and only if $L$ is fibered by $S_{\sigma}$.

Proof This is a immediate consequence of Corollary 1.

Lemma 2 Let $G_{\sigma}$ be a state graph and suppose there are two vertices $v$ and $w$ that decompose $G_{\sigma}$ into two connected components $X$ and $Y$, and there is an alternating path $\alpha$ from $v$ to $w$ in $Y$ that together with $X$ defines an inner cycle. (See Figure 8 .) Consider also the state surface $S_{y}$ induced by $\sigma$ and $Y$, and the state surface $S_{x}$ induced by $\sigma$ and $X \cup \alpha$. Then $S_{\sigma}$ is a fiber if and only if each surface, $S_{x}$ and $S_{y}$, is a fiber with respect to its boundary.

Proof Since $X \cup \alpha$ defines an inner cycle and $\alpha$ is alternating, with respect to the labels, then there is a ball $Q$ intersecting $S_{\sigma}$ at $S_{x}$ with the band associated with $\alpha$ in $\partial Q$. In this way, we can decompose $S_{\sigma}$ as a Murasugi sum of $S_{x}$ and $S_{y}$, as depicted in Figure 9.

From the result of Gabai and this Murasugi sum we have the statement of the lemma. 

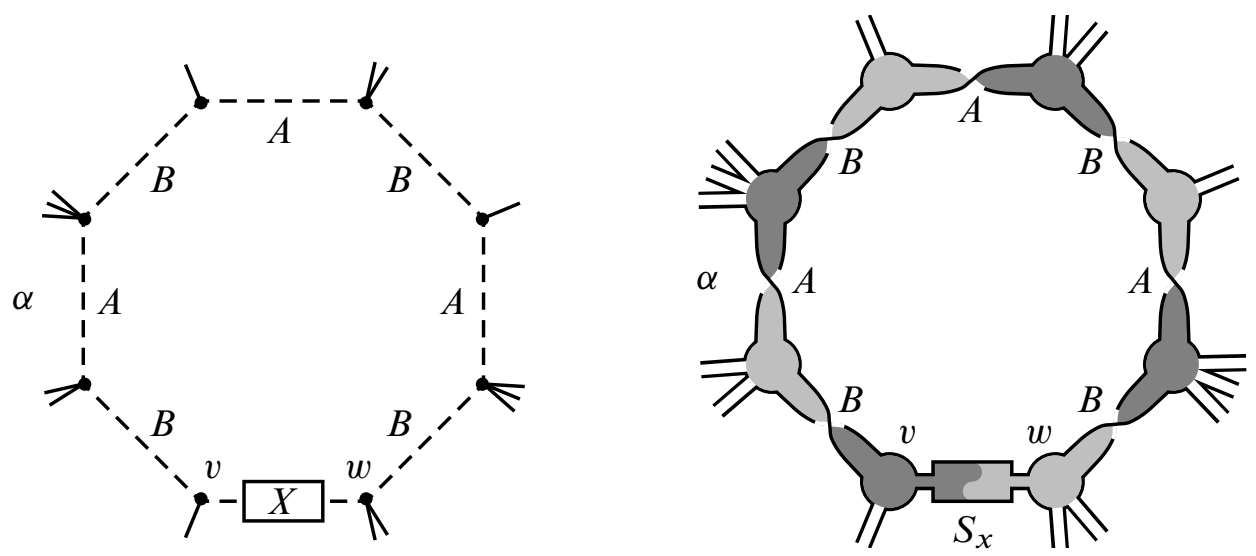

Figure 8: An alternating path $\alpha$ in that together with $X$ define an inner cycle
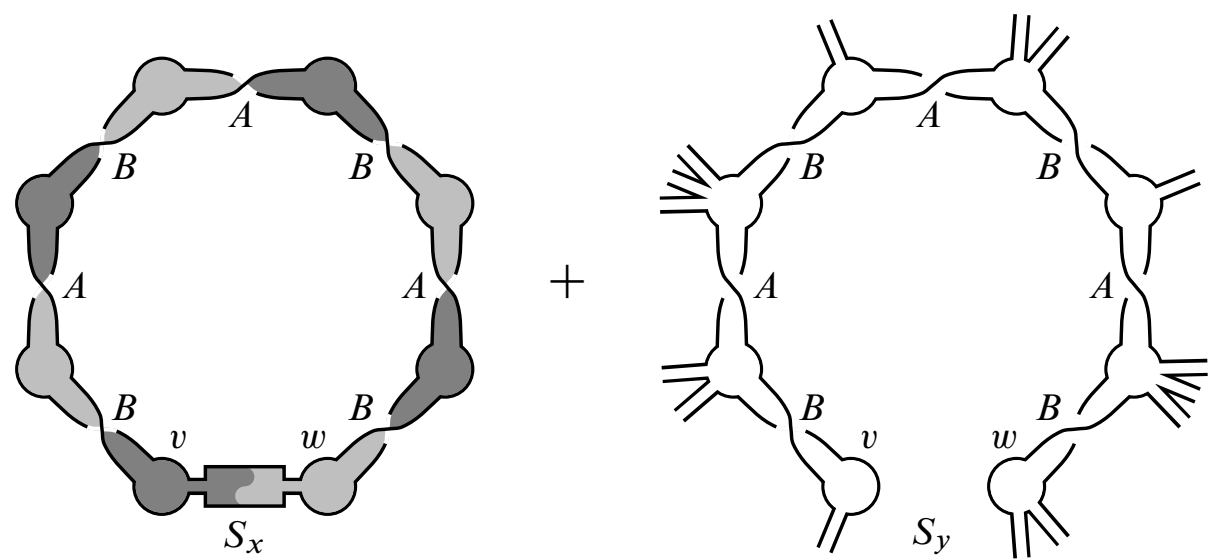

Figure 9: Decomposition of $S_{\sigma}$ by $\alpha$ as a Murasugi sum of $S_{x}$ and $S_{y}$

Lemma 3 If the state graph $G_{\sigma}$ has an inner cycle that is alternating with respect to the labels $A$ or $B$ then $S_{\sigma}$ is not a fiber of $L$.

Proof Consider an inner cycle $\gamma$ of $G_{\sigma}$. In Lemma 2, let $X$ be one edge of $\gamma$ and $\alpha$ the remaining edges. Then $S_{\sigma}$ is a fiber if and only if $S_{x}$ and $S_{y}$ are fibers. Since $\gamma$ is alternating then $S_{x}$ is an annulus, which is not a fiber of its boundary. Hence, $S_{\sigma}$ is not a fiber of $L$.

Proof of Theorem 1 We start by observing that if $G_{\sigma}^{\prime}$ is a tree then $S_{\sigma}^{\prime}$ is a disk, and hence a fiber of $L^{\prime}$. Therefore, by Corollary $2, L$ is fibered by $S_{\sigma}$. 
Suppose now that $G_{\sigma}^{\prime}$ has a cycle. Then $G_{\sigma}$ also has a cycle. Consider an inner cycle $\alpha$ of $G_{\sigma}$. Suppose there is a tree component to the interior of $\alpha$ at the common vertex. Then, using Lemma 1, we may decompose this tree. The surface induced by this tree is a disk, and hence a fiber for its boundary circle. Therefore, we may assume the following: consecutive edges in $\alpha$ are also consecutive in the common vertex, ie there are no edges to the interior of $\alpha$ between them. Given any two such edges in $\alpha$, since $\sigma$ is alternating they have different labels. Hence, $\alpha$ is alternating. Consequently, by Lemma 3 the state surface $S_{\sigma}$ is not a fiber of $L$.

\section{A new proof of Theorem 2}

In this section we present a different proof of Theorem 2. This theorem first appeared as [2, Theorem 5.21], but the proof presented there consists of a detailed study of polyhedral decompositions of $S^{3}-S_{\sigma}$. In [1], a much simpler proof is given: it is proved inductively via Murasugi sums together with Theorem 3 to deduce fibering information. Some of these ideas were also independently used in the work of the first author [4] and in the previous section. The proof we present is a consequence of Stallings' fibration criteria [8].

Theorem 4 (Stallings) Let $T \subset S^{3}$ be a compact, connected, oriented surface with nonempty boundary $\partial T$. Let $T \times[-1,1]$ be a regular neighborhood of $T$ and let $T^{+}=T \times\{1\} \subset S^{3}-T$. Let $f=\left.\varphi\right|_{T}$, where $\varphi: T \times[-1,1] \rightarrow T^{+}$is the projection map. Then $T$ is a fiber for the link $\partial T$ if and only if the induced map $f_{*}: \pi_{1}(T) \rightarrow \pi_{1}\left(S^{3}-T\right)$ is an isomorphism.

We describe the induced map in the case $T$ is the state surface associated to the reduced graph of a homogeneous link diagram. We will see that when $G_{\sigma}^{\prime}$ is a tree, the reduced surface $S_{\sigma}^{\prime}$ is a disk and the map $f_{*}$ is trivial, as desired. When $G_{\sigma}^{\prime}$ has cycles, we show that the map $f_{*}$ cannot be an isomorphism by showing that the corresponding map on first homology is not an isomorphism.

By using Lemma 1, we may decompose the reduced state graph $G_{\sigma}^{\prime}$ associated with the homogeneous link diagram along cut vertices. This has also been observed in [1, Lemma 3], where Futer proves that the reduced graph has no cut vertices if and only if it is an all- $A$ or all- $B$ state. Thus, we only need to prove this result for all- $A$ or all- $B$ states. We provide the proof for the case of an all- $A$ state, the other case being similar.

First note that in the absence of cut vertices in the graph $G_{\sigma}^{\prime}$, the surface $S_{\sigma}^{\prime}$ is a checkerboard surface. If the graph $G_{\sigma}^{\prime}$ is a tree, then the surface $S_{\sigma}^{\prime}$ is a disk. Hence $S_{\sigma}^{\prime}$ is a fiber, and by Corollary 2 the surface $S_{\sigma}$ is also a fiber. 
Suppose now that $G_{\sigma}^{\prime}$ is not a tree, ie that it has cycles. We will prove that this contradicts Stalling's theorem. First note that the fundamental group of the surface $S_{\sigma}^{\prime}$ is free. Consider the inner cycles $\alpha_{1}, \ldots, \alpha_{n}$ in $G_{\sigma}^{\prime}$ oriented in the counterclockwise direction. Since $S_{\sigma}$ is a fiber, it is orientable, hence $S_{\sigma}^{\prime}$ is also orientable and we choose a base point $a$ of $\pi_{1}\left(S_{\sigma}^{\prime}\right)$ such that, when seen from above the projecting plane, we see the base point $a$ in the " + " side of $S_{\sigma}^{\prime}$. Finally, add $\operatorname{arcs} h_{1}, \ldots, h_{n}$ from $a$ to each of the inner cycles above. This gives loops $\beta_{i}=h_{i} \alpha_{i} h_{i}^{-1}$ based at $a$. This set of based loops corresponds to a generating set for $\pi_{1}\left(S_{\sigma}^{\prime}\right)$. These generators will be denoted by $u_{1}, \ldots, u_{n}$.

Since the surface $S_{\sigma}^{\prime}$ is a checkerboard surface, its complement $S^{3}-S_{\sigma}^{\prime}$ also has a free fundamental group. We now describe a generating set for this group. There are two types of white regions in the projecting plane: one unbounded region and $n$ bounded ones, which correspond to the inner cycles of $G_{\sigma}^{\prime}$. Let $C_{0}$ denote the unbounded white region determined by $S_{\sigma}^{\prime}$ and let $A_{i}$ denote a white region determined by the inner cycle $\alpha_{i}$. Let $\gamma_{i} \subset S^{3}-S_{\sigma}^{\prime}$ be a semicircle with one endpoint in $C_{0}$ and the other in $A_{i}$, lying under the projecting plane. Let $f: S_{\sigma}^{\prime} \rightarrow S^{3}-S_{\sigma}^{\prime}$ be the function described in Theorem 4. Associated to each region $A_{i}$ we construct a simple closed curve by connecting the endpoints of the arc $\gamma_{i}$ to the point $f(a)$ by straight line segments. Each of these curves is oriented so that, starting at $f(a)$, we move along the line segment connecting $f(a)$ to the endpoint of $\gamma_{i}$ in $A_{i}$, then move along $\gamma_{i}$ to the second endpoint and then back to $f(a)$ through the second line segment. We have built loops with base point $f(a)$ corresponding to a set of generators for $\pi_{1}\left(S^{3}-S_{\sigma}^{\prime}\right)$. These generators are denoted by $x_{1}, \ldots, x_{n}$, according to the label of region they cross.

Let $S_{\sigma}^{\prime+}$ be the copy of $S_{\sigma}^{\prime}$ in $S^{3}-S_{\sigma}^{\prime}$ parallel to $S_{\sigma}^{\prime}$, obtained from $S_{\sigma}^{\prime}$ by pushing it in the "+" direction. This is formally defined by the map $f: S_{\sigma}^{\prime} \rightarrow S^{3}-S_{\sigma}^{\prime}$ described in Theorem 4. The induced map $f_{*}$ can be described by determining the image of each generator $u_{i} \in \pi_{1}\left(S_{\sigma}^{\prime}\right)$. We write $f_{*}\left(u_{i}\right)$ as a word on the generators $x_{1}, \ldots, x_{n}$, given by the image the loop $\beta_{i}=h_{i} \alpha_{i} h_{i}^{-1}$,

$$
f_{*}\left(u_{i}\right)=w_{h_{i}} w_{\alpha_{i}} w_{h_{i}}^{-1},
$$

where $w_{h_{i}}$ is the word on the letters $x_{1}, \ldots, x_{n}$ given by the image of the arcs $h_{i}$ under the map $f$. The word $w_{\alpha_{i}}$ is obtained by the image of the cycle $\alpha_{i}$ as follows. Suppose that $\alpha_{i}$ and $\alpha_{j}$ have a common edge. Vertices are labeled "+" or "-_, depending on the side of the surface they lie. We have two possibilities:

Case 1 The orientation induced on the edge by $\alpha_{i}$ is from a "+" vertex to a "-" vertex. In this case we write the letter $x_{i}$. (See Figure 10 left.) 
Case 2 The orientation induced on the edge by $\alpha_{i}$ is from a "-" vertex to a "+" vertex. In this case we write the letter $x_{j}^{-1}$. (See Figure 10 right.)
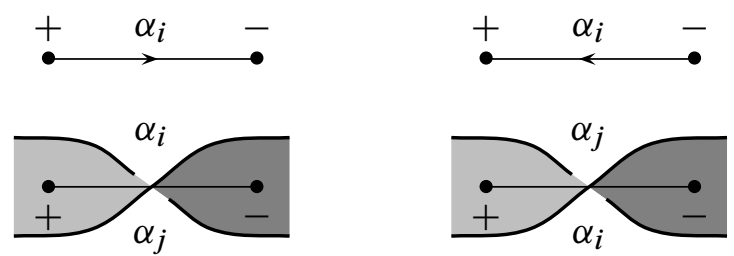

Figure 10: Left: Case 1; right: Case 2

Remark 2 It is important to notice the inner cycle $\alpha_{i}$ may share an edge with the unbounded region $C_{0}$. If this is the case, in 2 above, we write no letters corresponding to this edge.

Remark 3 Observe that the loops $\alpha_{i}$ and $\alpha_{j}$ induce reverse orientations on the edges they share. Therefore, when we write the letters corresponding to the loop $\alpha_{j}$, the letter corresponding to this edge is the same letter as $\alpha_{i}$, with opposite sign, ie either $x_{i}^{-1}$ or $x_{j}$. This is illustrated in Figure 10 .

Now we consider the map $\overline{f_{*}}: H_{1}\left(S_{\sigma}^{\prime}\right) \rightarrow H_{1}\left(S^{3}-S_{\sigma}^{\prime}\right)$ induced on homology by $f_{*}$. Denote by $\bar{u}_{1}, \ldots, \bar{u}_{n}$ the generators of $H_{1}\left(S_{\sigma}^{\prime}\right)$, corresponding to the generators of $\pi_{1}\left(S_{\sigma}^{\prime}\right)$. The generators of $H_{1}\left(S^{3}-S_{\sigma}^{\prime}\right)$ are defined similarly and denoted $\bar{x}_{1}, \ldots, \bar{x}_{n}$. The map $\overline{f_{*}}$ is given by a $n \times n$ matrix $\mathcal{A}=\left[a_{i j}\right]$, where the $i^{\text {th }}$ column is the vector $\bar{f}\left(\bar{u}_{i}\right) \in H_{1}\left(S^{3}-S_{\sigma}^{\prime}\right)$. By the description of the map $f_{*}$ and the remarks above, the matrix $\mathcal{A}$ has the following properties:
(i) $a_{i i} \geq 2$,
(ii) $a_{i i} \geq \sum_{j \neq i}\left|a_{i j}\right|$
(iii) $a_{i i} \geq \sum_{j \neq i}\left|a_{j i}\right|$

(i) follows from the fact that every inner cycle in $G_{\sigma}^{\prime}$ has at least 4 edges; (ii) and (iii) follow from the fact that, when we go through the cycle $\alpha_{i}$, at every other edge we write the letter $x_{i}$ and at the remaining edges we write one of the other letters $x_{j}$ or write no letters (as in Remark 3).

To prove that the map $f_{*}$ is not an isomorphism if $G_{\sigma}^{\prime}$ is not a tree (ie has cycles), it suffices to prove the matrix $\mathcal{A}$ is not invertible over $\mathbb{Z}$. This is straightforward by the following theorem.

Theorem 5 Let $\mathcal{A}=\left[a_{i j}\right]$ be such that $a_{i i} \geq \max \left(2, \sum_{j \neq i}\left|a_{i j}\right|\right)$ for all $i \in\{1, \ldots, n\}$. If $\operatorname{det}(\mathcal{A}) \neq 0$, then $\operatorname{det}(\mathcal{A}) \geq 2$ and this inequality is sharp. 
Proof We will prove the theorem by induction on $n$.

For $n=1, \operatorname{det}(\mathcal{A})=\operatorname{det}\left[a_{11}\right]=a_{11} \geq 2$.

Consider now any $n \in \mathbb{N}$ and suppose that the result is true for $n-1$. Suppose $\operatorname{det}(\mathcal{A}) \neq 0$ and let $\mathcal{B}=\left[b_{i j}\right] \in M_{n}(\mathbb{Z})$ be the adjugate matrix of $\mathcal{A}$. Then $\mathcal{A B}=(\operatorname{det} \mathcal{A}) \mathcal{I}_{n}$.

If all elements of the column $j$ of $\mathcal{B}$ have the same absolute value $b_{j j}$, then $\operatorname{det}(\mathcal{A})=\sum_{k} a_{i j} b_{j i}$ is a multiple of $b_{j j} \geq 2$.

If not, suppose $\left|b_{i j}\right| \geq\left|b_{k j}\right|$ for all $k \in\{1, \ldots, n\}$ and $\left|b_{i j}\right|>\left|b_{k j}\right|$ for some $k$. Then

$\left|\sum_{k=1}^{n} a_{i k} b_{k j}\right|=\left|a_{i i} b_{i j}+\sum_{k \neq i} a_{i k} b_{k j}\right| \geq\left|a_{i i} b_{i j}\right|-\sum_{k \neq i}\left|a_{i k} b_{k j}\right|>\left|a_{i i} b_{i j}\right|-\left|a_{i i} b_{i j}\right|=0$.

Since $\mathcal{A B}$ is a diagonal matrix, then $i=j$. Therefore, $\left|b_{i i}\right|>\left|b_{k i}\right|$, for all $k \neq i$. Furthermore, by the induction hypothesis, $b_{i i} \geq 2$. Hence

$$
\begin{aligned}
\operatorname{det}(\mathcal{A})=\left|\sum_{k=1}^{n} a_{i k} b_{k i}\right|=\left|a_{i i} b_{i i}+\sum_{k \neq i} a_{i k} b_{k i}\right| & \geq\left|a_{i i} b_{i i}\right|-\sum_{k \neq i}\left|a_{i k} b_{k i}\right| \\
& \geq a_{i i} b_{i i}-a_{i i}\left(b_{i i}-1\right)=a_{i i} \geq 2 .
\end{aligned}
$$

To see that the inequality is sharp, observe that the determinant of the $n \times n$ matrix

$$
\left[\begin{array}{rrrrrrrr}
2 & 2 & 0 & 0 & 0 & \cdots & 0 & 0 \\
1 & 2 & -1 & 0 & 0 & \cdots & 0 & 0 \\
1 & 0 & 2 & 1 & 0 & \cdots & 0 & 0 \\
0 & 0 & 1 & 2 & 1 & \cdots & 0 & 0 \\
\vdots & \vdots & \vdots & \vdots & \vdots & \ddots & \vdots & \vdots \\
0 & 0 & 0 & 0 & 0 & \cdots & 2 & 1 \\
0 & 0 & 0 & 0 & 0 & \cdots & 1 & 2
\end{array}\right]
$$

is 2 for every $n \in \mathbb{N}$.

Acknowledgements The first author was partially supported by FUNCAP (project PJP-0072-00013.01.00/12) and CNPq (project Universal).

This work was partially developed while the second author was visiting the Department of Mathematics at Federal University of Ceará. He is thankful for the support to this visit by the host institution and also for the warm hospitality. 
The second and third authors were partially supported by the Centro de Matemática da Universidade de Coimbra (CMUC), funded by the European Regional Development Fund through the program COMPETE and by the Portuguese Government through the FCT - Fundação para a Ciência e a Tecnologia under the project PEstC/MAT/UI0324/2011.

\section{References}

[1] D Futer, Fiber detection for state surfaces, Algebr. Geom. Topol. 13 (2013) 2799-2807 MR3116303

[2] D Futer, E Kalfagianni, J Purcell, Guts of surfaces and the colored Jones polynomial, Lecture Notes in Mathematics 2069, Springer, Heidelberg (2013) MR3024600

[3] D Gabai, Detecting fibred links in $S^{3}$, Comment. Math. Helv. 61 (1986) 519-555 MR870705

[4] D Girão, On the fibration of augmented link complements, Geom. Dedicata 168 (2014) 207-220 MR3158039

[5] S Goodman, G Tavares, Pretzel-fibered links, Bol. Soc. Brasil. Mat. 15 (1984) 85-96 MR794730

[6] J Harer, How to construct all fibered knots and links, Topology 21 (1982) 263-280 MR649758

[7] K Murasugi, On a certain subgroup of the group of an alternating link, Amer. J. Math. 85 (1963) 544-550 MR0157375

[8] J R Stallings, Constructions of fibred knots and links, from: "Algebraic and geometric topology, Part 2", (R J Milgram, editor), Proc. Sympos. Pure Math. 32, Amer. Math. Soc. (1978) 55-60 MR520522

DG: Department of Mathematics, Universidade Federal do Ceará Av. Humberto Monte S/N, Campus do Pici - Bloco 914, 60455-760 Fortaleza-CE, Brazil JN, AS: CMUC, Department of Mathematics, University of Coimbra Apartado 3008, EC Santa Cruz, 3001-501 Coimbra, Portugal

dgirao@mat.ufc.br, nogueira@mat.uc.pt, ams@mat.uc.pt

Received: 25 June 2014 Revised: 1 October 2014 
